\title{
Novel and Classic Myoepithelial/Stem Cell Markers in Metaplastic Carcinomas of the Breast
}

\author{
Jorge S. Reis-Filho, M.D., ${ }^{* \dagger}$ Fernanda Milanezi, M.D., ${ }^{*}$ \\ Joana Paredes, B.Sc., ${ }^{*}$ Paula Silva, B.T., ${ }^{*}+$ Emílio M. Pereira, M.D.,§ \\ Sueli A. Maeda, M.D.,§ Leda V. de Carvalho, M.D.,§, and \\ Fernando C. Schmitt, M.D., Ph.D.* $*$
}

Metaplastic carcinomas of the breast (MCBs) are unusual neoplasms characterized by an admixture of glandular epithelial components, which frequently exhibit features of squamous differentiation, and mesenchymal malignant components. Regardless of the presence of myoepithelial features in MCB, no consensus concerning their putative histogenesis has yet been achieved. Recently, novel putative myoepithelial markers have been developed, including p63, maspin, and P-cadherin. We assessed the expression of these myoepithelial markers in MCBs and compared their expression with classic myoepithelial markers. Immunohistochemistry using the streptavidinbiotin-peroxidase complex technique with antibodies raised against p63, maspin, P-cadherin, actin (clones CGA7, 1A4 and HHF35), cytokeratin 14 (Ck14), and vimentin was performed on 16 MCBs (7 matrix-producing MCBs, 6 adenosquamous MCBs, and 3 MCBs with heterologous elements). In healthy breast lobules and ducts adjacent to the tumors, myoepithelial cells showed distinctive and consistent immunoreactivity for p63, maspin, P-cadherin, actin, S-100 protein, and Ck14. Matrix-producing MCBs were positive for maspin in all cases, for p63 in 4 of 7 cases, and for P-cadherin in 4 of 7 cases. Adenosquamous MCB showed immunoreactivity for p63, maspin, and P-cadherin in 5 of 6 cases. All novel myoepithelial markers and $\mathrm{Ck} 14$ decorated squamous cell islands. MCBs with heterologous elements were positive for p63 in 1 case, for maspin in all 3 cases, and for P-cadherin in 2 cases. All cases showed at least one of the novel myoepithelial markers. Eleven of 16 cases were positive for actin. Eleven of 14 cases reacted with $\mathrm{Ck} 14$, and all cases that stained for S-100 protein (9 of 9) and

Manuscript received June 10, 2002; accepted September 4, 2002.

From the *Institute of Molecular Pathology and Immunology (IPATIMUP) and $\ddagger$ Medical Faculty, University of Porto, Porto, Portugal; $†$ School of Health Sciences, University of Minho, Braga, Portugal; and §Salomão and Zoppi Associated Pathologists, São Paulo, Brazil.

This study was partially supported by a Ph.D. grant from the Portuguese Science and Technology Foundation (Fundação para a Ciência e a Tecnologia [FCT], Ministério da Ciência e Tecnologia [MCT], Portugal, reference SFRH/BD/5386/2001).

This is an original study partially presented as a poster at the meeting of the United States and Canadian Academy of Pathology, Chicago, IL, February 23-March 2, 2002. The abstract was published as follows: Reis-Filho JS, Milanezi F, Paredes J, et al. Maspin, p63 and p-cadherin expression in metaplastic breast carcinomas: new insights into a putative myoepithelial histogenesis differentiation. Mod Pathol 2002;15: 191. Reis-Filho JS, Milanezi F, Paredes J, et al. Maspin, p63 and p-cadherin expression in metaplastic breast carcinomas: new insights into a putative myoepithelial histogenesis differentiation. Laboratory Invest 2002;82:191.

Address correspondence and reprint requests to Jorge S. Reis-Filho, M.D., IPATIMUP, R. Roberto Frias, S/N, 4200 Porto, Portugal. Email: jreis@ipatimup.pt vimentin (13 of 13) were also positive. Based on our findings, the balance of probabilities favors that MCBs may have a basal or myoepithelial cell histogenesis and differentiation.

Key Words: Breast-Carcinosarcoma-Immunohistochemistry-Matrix-producing carcinoma-Metaplastic carcinomaMyoepithelium-Spindle cell carcinoma.

Applied Immunohistochemistry \& Molecular Morphology 11(1): 1-8, 2003.

Metaplastic carcinoma of the breast (MCB) is an umbrella descriptive term that encompasses several types of breast carcinomas that show the presence of peculiar metaplastic elements (1-10). Since its first description, several efforts have been driven to characterize its histogenesis, but most, if not all, of them fell short in achieving this goal (1-10).

Recently, Perou et al. (11), using cDNA microarray technology, described four distinctive patterns of gene expression clustering in breast carcinomas, by which they may be classified in four distinct gene expression patterns: luminal epithelial and estrogen receptorpositive pattern; normal breastlike pattern; c-erb-B2positive pattern; and basal cell and myoepithelial cell pattern. Later, this research group (12) showed a correlation between basal and c-erb-B2-positive groups with an aggressive biologic behavior.

Because MCB shows mesenchymal-like spindleshaped cells or metaplastic elements, including bone, cartilage, and squamous cells (1-10), and may genetically belong to the basal cell and myoepithelial cell pattern, we attempted to evaluate the expression of maspin, p63, and P-cadherin, which are molecules consistently expressed by breast myoepithelial and basal (stem) cells.

Maspin, also known as mammary serpin, is a 42 kilodalton protein that belongs to the serine protease inhibitor superfamily and presents an important role in mammary development (13-16). It has been suggested that maspin possesses tumor growth suppression, tumor invasiveness suppression, and antiangiogenic properties (13-16). In healthy breast tissue, its expression is almost confined to the myoepithelial and stem cell compartments of ducts and terminal ductulobular units (TDLUs) (15-16). Recently, our group described the consistent 
expression of maspin in myoepithelial cell tumors of the breast (16). Conversely, Lele et al. (15) and Maass et al. (14) showed that a minor proportion of tubular and other invasive carcinomas of the breast express maspin. To the best of our knowledge, no systematic evaluation of maspin expression in MCB has hitherto been performed.

p63 is a recently described p53 homolog nuclear transcription factor that is necessary for mammary development, as shown in knockout mouse models $(17,18)$. The TP63 gene encodes at least 6 distinct isoforms that harbor trans-activating (TAp63) or dominant-negative $(\Delta \mathrm{Np} 63)$ activities on the p53 reporter genes $(17,18)$. The $\Delta \mathrm{Np} 63$ is consistently expressed in the basal and stem cell population of stratified epithelia and is thought to be necessary for the maintenance of a somatic stem cell population in these tissues $(17,18)$. Recently, Barbareschi et al. (18) evaluated $\Delta \mathrm{Np} 63$ and TAp63 expression in healthy breast tissue and human breast cancers. Remarkably, they observed that $\Delta \mathrm{Np} 63$ was expressed in the myoepithelial and the basal and stem cell compartment of healthy breast and only rarely expressed by invasive carcinomas and their metastases (18).

$\mathrm{P}$-cadherin is a calcium-dependent glycoprotein that plays a major role in homotypic-homophilic cell adhesion (19-24). This molecule possesses an intriguing distribution in human epithelial cells, being restricted to basal and stem cells of stratified epithelia, such as epidermis and urothelium (19-24). In breast ducts and TDLU, P-cadherin is confined to the membranes of myoepithelial and stem (basal) cells (19-24). Moreover, several studies have pointed out a remarkable association of P-cadherin expression in human breast carcinomas and an embryonic myoepithelial and stem cell-like phenotype $(20,21,23)$, which seems to be similar to the myoepithelial-basal-stem cell pattern described by Perou et al. (11) and Sorlie et al. (12).

To analyze a putative myoepithelial and basal (stem) cell histogenesis of MCB, we assessed the immunohistochemical expression of these three myoepithelial and stem cell markers in 16 bona fide cases of MCB and compared them with classic myoepithelial markers, $\alpha$-smooth muscle actin (ASMA), muscle-specific actin (MSA), S-100 protein, cytokeratin 14 (Ck14), and vimentin, which are variably, but consistently, expressed in myoepithelial cell neoplasms.

\section{MATERIAL AND METHODS}

Sixteen cases of MCBs were retrieved from the consultation files of two of the authors (E.M.P., F.C.S.). The clinical pathologic information was obtained from the surgical pathology reports and by contacting the referring pathologists.

Automated immunohistochemistry (Labvision Autostainer LV-1) according to the streptavidin-biotin- peroxidase techniques using antibodies raised against maspin (clone EAW24, 1:50, Novocastra, Newcastle, United Kingdom), p63 (clone 4A4, 1:150, Neomarkers, Freemont, CA), P-cadherin (clone 56, 1:25, Transduction, Lexington, KY), MSA (clone HHF35, 1:50, DAKO Corp., Carpinteria, CA), ASMA (clone CGA7, 1:5, Enzo Diagnostics, New York, NY and clone 1A4, 1/1600, DAKO Corp.), S-100 protein (polyclonal, 1:10,000, DAKO Corp.), Ck14 (clone LL002, prediluted, Serotec Ltd., Oxford, United Kingdom), and vimentin (clone V9, 1:400, DAKO Corp.) were performed on $4-\mu \mathrm{m}$ sections. Previous heat-induced antigen retrieval with DAKO antigen retrieval solution was performed. Positive and negative controls were included in each slide run. All controls gave satisfactory results. In addition, in all samples, healthy breast lobules and ducts were available as internal controls. Briefly, in nonneoplastic breast tissue, maspin should stain the nuclei and cytoplasm of myoepithelial cells of breast lobules and ducts (25). p63 should show nuclear positivity in myoepithelial cells of nonneoplastic breast lobules and ducts. P-cadherin should present a distinctive membranous and occasionally cytoplasmic immunoreactivity in nonneoplastic myoepithelial cells. MSA and ASMA should decorate myoepithelial cells, vessel walls, and scattered stromal cells. Cytokeratin 14 should stain myoepithelial cells of the breast lobules and ducts.

Because nonneoplastic mammary secretory cells do not express P-cadherin, either membranous or cytoplasmic, P-cadherin immunoreactivity was considered positive when more than $5 \%$ of the neoplastic cells expressed this marker $(18,22)$. Similarly, we adopted the same cutoff value for nuclear and cytoplasmic maspin reactivity, nuclear p63 reactivity, and cytoplasmic ASMA, MAS, Ck14, and vimentin reactivity.

\section{RESULTS}

\section{Clinical and Pathologic Findings}

All patients were women, and their ages ranged from 43 to 78 years (mean, 61 years). Tumor size ranged from 0.9 to $6.5 \mathrm{~cm}$ (mean, $3.32 \mathrm{~cm}$; median, $3.25 \mathrm{~cm}$ ). All cases were classified according to current criteria for histologic classification of metaplastic breast carcinoma $(1,4-10)$. Seven cases showed the prototypical histologic appearance of matrix-producing MCB (Fig. 1A). Three cases were adenosquamous carcinoma of the breast (Fig. 1E). Three cases were spindle cell carcinomas of the breast with rare to occasional squamous cell components (two low and one high grade) (Fig. 2A), and three cases were high grade breast carcinomas with heterologous elements (Fig. 2E). Among the last three cases, Case 14 was composed of papillary projections lined by proliferated myoepithelial and secretory cells, sometimes arranged in tubulelike and papillary structures, admixed 

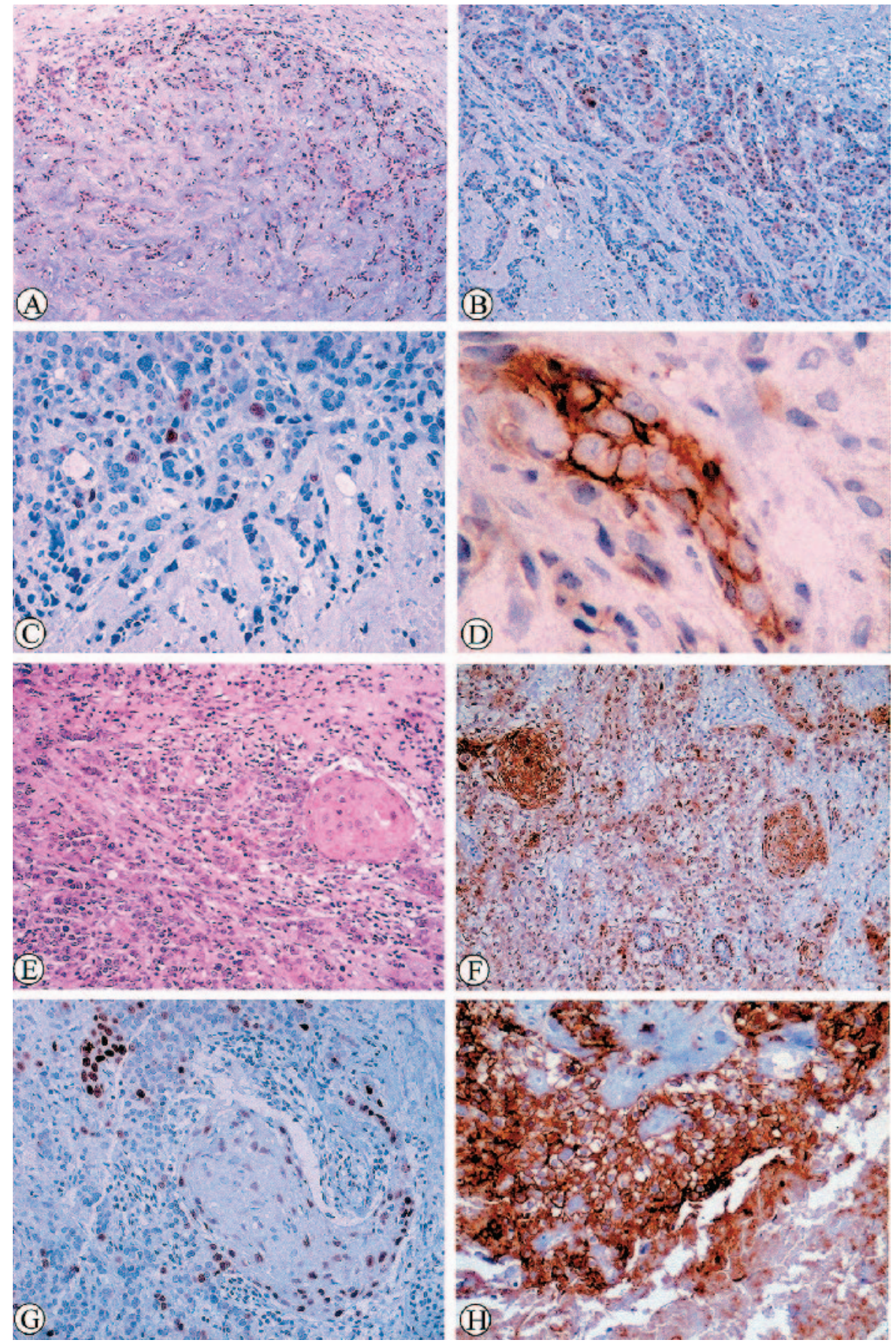

FIG. 1. Matrix-producing metaplastic carcinoma of the breast (A-D). A: Medium-power magnification of a matrix producing metaplastic carcinoma of the breast. B: Maspin immunoreactivity in the epithelioid cells. C: Focal p63 expression in the nuclei of neoplastic cells. D: Strong cytoplasmic reactivity for P-cadherin in neoplastic cells. High grade adenosquamous metaplastic carcinoma of the breast (E-H). E: Medium-power magnification of a high grade adenosquamous carcinoma of the breast. Note the presence of a focus of squamous differentiation on the right. F: Maspin positivity in the nuclei and cytoplasm of neoplastic cells. Note that maspin decorated myoepithelial cells in "normal" lobules on the lower center. G: p63 expression in neoplastic cells of the typical carcinomatous component and in the squamous cell component. Note that some of the nuclei of the terminally differentiated squamous cells fail to highlight for p63. H: Neoplastic cells strongly stained with $\mathrm{P}$-cadherin in a membranous and cytoplasmic distribution $(A, E$, hematoxylin and eosin; B-D, F-H, streptavidinbiotin-peroxidase and DAB). with atypical spindle cells arranged in a patternless fashion, bone trabeculae, and chondroid foci. Case 15 showed an admixture of high grade invasive ductal carcinoma, squamous cell islands, chondroid areas, and scattered highly pleomorphic cells. Case 16 was a heterogeneous neoplasm, composed of high grade solid epithelial areas, chondroid foci, and a large amount of pleomorphic multinucleated anaplastic cells. Table 1 summarizes the clinical pathologic parameters of the patients.

\section{Immunohistochemical Findings}

All cases were positive for at least one myoepithelial marker. Table 2 summarizes the immunohistochemical findings.

\section{Maspin}

In the healthy myoepithelial cells of adjacent breast lobules and ducts, maspin stained the nucleus and cytoplasm of myoepithelial cells. Nuclear or cytoplasmic 


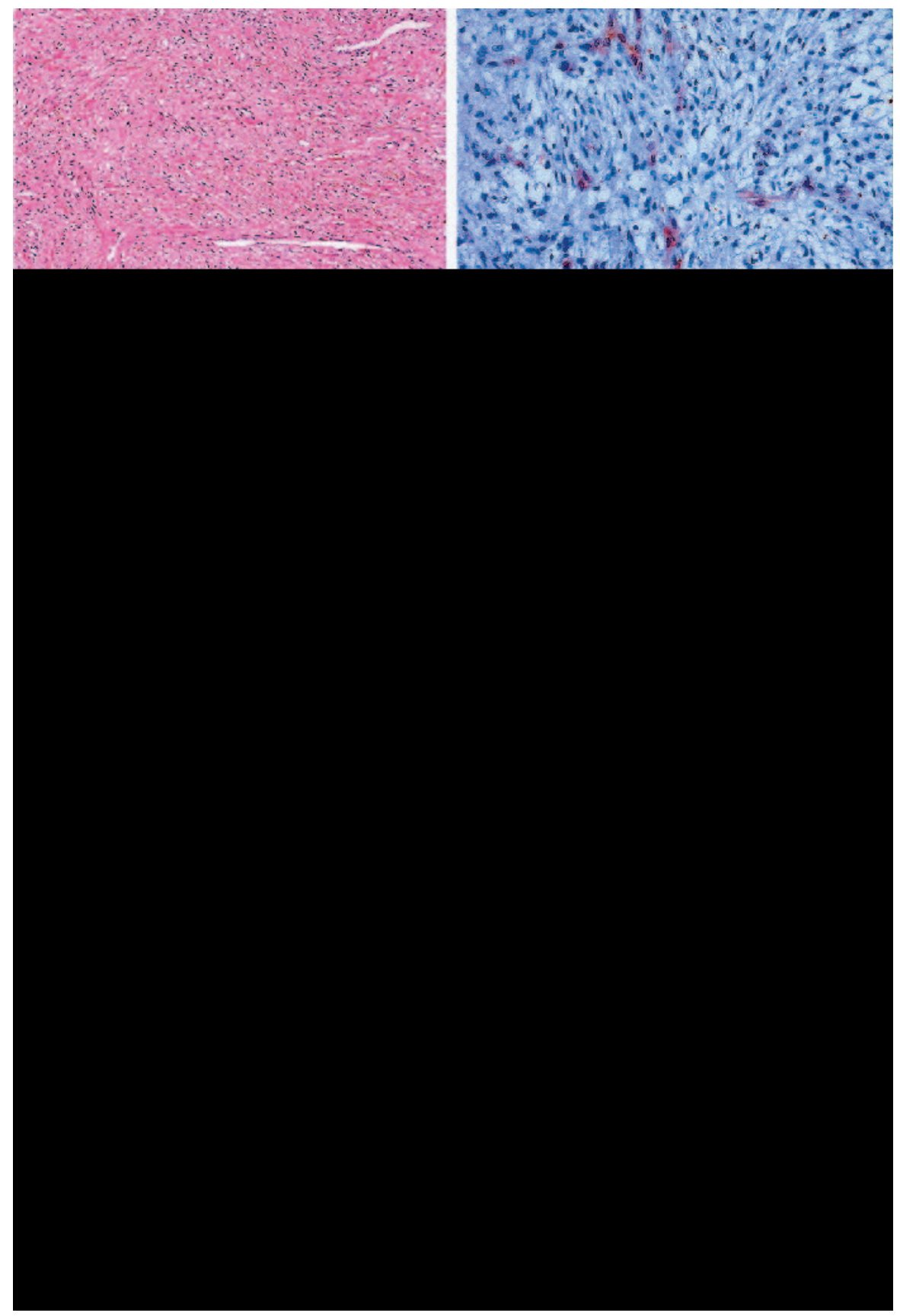

FIG. 2. Low grade spindle cell metaplastic carcinoma of the breast (A-D). A: Medium power magnification of a low grade spindle cell carcinoma of the breast. B: Cytoplasmic maspin expression in the cytoplasm of the epithelioid cells arranged in cords and strands. C: p63 expression in the nuclei of epithelioid cells. D: Cytoplasmic and membranous P-cadherin expression in cells with similar morphology and distribution. Carcinosarcoma (metaplastic carcinoma with heterologous elements) of the breast (E-H). E: Low-power view illustrating the complex admixture of tubulopapillary structures and metaplastic elements. F: Maspin expression in the cells arranged in papillary structures. G: These same cells were also decorated with p63 antibody. Note on the left side the presence of "normal" ducts with p63positive myoepithelial cells. H: Pcadherin expression in tubulopapillary structures $(A, E$, hematoxylin and eosin; B-D, F-H, streptavidin-biotin-peroxidase and $\mathrm{DAB}$ ).

maspin expression was observed in 15 of $16(93.75 \%)$ MCBs; a concurrent nuclear and cytoplasmic staining was found in 12 cases. In three additional cases, only a cytoplasmic immunoreactivity pattern was highlighted. Maspin immunoreactivity was detected in all seven matrix-producing MCBs (Fig. 1B), in all three adenosquamous MCBs (Fig. 1F), in two of three (66.67\%) spindle cell MCBs (Fig. 2B), and in all three MCBs with heterologous elements (Fig. 2F). In one spindle cell MCB, one matrix-producing $\mathrm{MCB}$, and one $\mathrm{MCB}$ with heterologous elements, maspin was restricted to the cytoplasm of the neoplastic cells. It is noteworthy that maspin was also observed in most of the squamous cell differentiation foci (Cases 1, 2, 4-6, and 15).

p63

In all control areas, p63 expression was observed in the nucleus of myoepithelial cells of adjacent nonneoplastic breast lobules and ducts. p63 was observed in the nuclei of neoplastic cells in 10 of $16(62.5 \%) \mathrm{MCBs}$, distributed as such: 4 of 7 (57.14\%) matrix-producing MCBs (Fig. 1C), 2 of 3 (66.67\%) adenosquamous MCBs (Fig. 1G), 3 of 3 (100\%) spindle cell MCBs (Fig. 2C), and 1 of $3(33.33 \%)$ MCBs with heterologous elements 
TABLE 1. Clinicopathological findings of metaplastic carcinoma of the breast

\begin{tabular}{cccc}
\hline Case & Age & $\begin{array}{c}\text { Size } \\
(\mathrm{cm})\end{array}$ & $\begin{array}{c}\text { Nuclear } \\
\text { grade }\end{array}$ \\
\hline 1 & 71 & 3.5 & 2 \\
2 & 78 & 4.2 & 3 \\
3 & 64 & 3.3 & 3 \\
4 & 43 & 4.5 & 2 \\
5 & 74 & 6.5 & 2 \\
6 & 66 & 3.1 & 2 \\
7 & 43 & 3.2 & 3 \\
8 & 68 & 2.1 & 3 \\
9 & 75 & 2 & 3 \\
10 & N.A. & 0.9 & 3 \\
11 & 44 & 3 & 2 \\
12 & 43 & 1.5 & 3 \\
13 & 74 & 3.6 & 3 \\
14 & 75 & 3.8 & 2 \\
15 & 52 & 5 & 3 \\
16 & 45 & 2.9 & 3 \\
\hline
\end{tabular}

N.A., not available.

(33.33\%) (Fig. 2G). Nuclear expression of p63 was detected in the foci of squamous cell differentiation (Cases 1,2 , and 4-6).

\section{P-cadherin}

In adjacent control breast lobules and ducts, Pcadherin highlighted the membrane of myoepithelial cells. P-cadherin cytoplasmic or membranous immunoreactivity was observed in 11 of 16 cases, including 4 of $7(57.14 \%)$ matrix-producing MCBs (Fig. 1D), 3 of 3 (100\%) adenosquamous MCBs (Fig. 1H), 2 of 3 (66.67\%) spindle cell MCBs (Fig. 2D), and 2 of 3 (66.67\%) MCBs with heterologous elements (Fig. 2H).
In five cases (Cases 1-4 and 6), cytoplasmic expression of P-cadherin was highlighted in squamous cell nests.

\section{Classic Myoepithelial Markers}

For classic myoepithelial markers, ASMA and MSA decorated myoepithelial cells of breast lobules, ducts, and vessel walls and showed immunoreactivity in scattered stromal cells. S-100 protein showed strong nuclear and cytoplasmic positivity in myoepithelial cells, nerve bundles, scattered stromal cells, and some epithelial secretory cells. Ck14 consistently decorated the cytoplasm of myoepithelial cells of ducts but also showed a variable reactivity in lobules. ASMA showed divergent results according to the different clones we used in the current study. Whereas 6 of 8 cases showed rather remarkable immunoreactivity for 1A4 clone (3 matrix-producing MCBs, 2 spindle cell MCBs, and $1 \mathrm{MCB}$ with heterologous elements), 8 of 16 cases were immunoreactive for clone CGA7 (3 matrix-producing MCBs, 1 high grade adenosquamous carcinoma, 2 low grade spindle cell carcinomas with squamous cells, and $2 \mathrm{MCB}$ with heterologous elements). MSA was positive in all cases evaluated (one high grade spindle cell MCB with rare squamous cells, one low grade spindle cell MCB with rare squamous cells, and four matrix-producing MCBs). Nine cases were evaluated for S-100 protein immunoreactivity; in all cases, S-100 positivity was observed (two low grade adenocarcinomas with squamous cells, five matrix-producing MCBs, and two MCBs with heterologous elements). Ck14 immunoreactivity was assessed in 14 cases; in 11 cases, a rather strong and multifocal Ck14 expression was found. Four of five matrix-producing MCBs, three of four high grade spindle cell carcinomas,

TABLE 2. Expression of myoepithelial cell markers in metaplastic carcinomas of the breast

\begin{tabular}{|c|c|c|c|c|c|c|c|c|c|c|c|}
\hline \multirow[t]{2}{*}{ Case } & \multirow[t]{2}{*}{ Histological type } & \multirow[t]{2}{*}{ P63 } & \multicolumn{2}{|c|}{ Maspin } & \multirow[t]{2}{*}{ P-Cad } & \multirow[t]{2}{*}{$\mathrm{ASMA}^{*}$} & \multirow[t]{2}{*}{$\mathrm{ASMA}^{* *}$} & \multirow[t]{2}{*}{ SMA } & \multirow[t]{2}{*}{ S-100 } & \multirow[t]{2}{*}{ CK14 } & \multirow[t]{2}{*}{ Vim } \\
\hline & & & $\mathrm{N}$ & C & & & & & & & \\
\hline 1 & High grade Adenosquamous MCB & + & + & + & + & - & N.A. & N.A. & N.A. & - & N.A. \\
\hline 3 & High grade adenosquamous MCB & - & + & + & + & - & N.A. & N.A. & N.A. & + & ++ \\
\hline 4 & High grade spindle cell MCB (rare squamous cells) & + & + & + & + & - & N.A. & + & N.A. & + & ++ \\
\hline 5 & Low-grade spindle cell MCB (rare squamous cells) & + & - & - & - & + & + & + & + & + & + \\
\hline 6 & Low-grade spindle cell MCB (rare squamous cells) & + & - & + & + & + & + & N.A. & + & + & + \\
\hline 7 & Matrix producing MCB & + & + & + & - & + & N.A. & N.A. & N.A. & + & + \\
\hline 8 & Matrix producing MCB & + & + & + & + & - & - & N.A. & + & N.A. & + \\
\hline 9 & Matrix producing MCB & - & + & + & + & - & - & + & + & - & + \\
\hline 10 & Matrix producing MCB & - & - & + & - & - & + & N.A. & + & + & + \\
\hline 11 & Matrix producing MCB & + & + & + & - & - & N.A. & + & N.A. & N.A. & N.A. \\
\hline 12 & Matrix producing MCB & - & + & + & + & + & + & + & + & + & + \\
\hline 13 & Matrix producing MCB & + & + & + & + & + & + & + & + & + & + \\
\hline 14 & MCB with heterologous elements & + & + & + & + & + & + & N.A. & + & + & + \\
\hline
\end{tabular}

ASMA, $\alpha$-smooth muscle actin; C, cytoplasmic; CK14, Cytokeratin 14; MCB, metaplastic carcinoma of the breast; MSA, muscle specific actin; N, nuclear; N.A., not available; P-Cad, P-cadherin; SMA, smooth muscle actin; Vim, vimentin.

*: Enzo Diagnostics, clone CGA7, 1:5

**: DAKO, clone $1 \mathrm{~A} 4,1 / 1600$

Note: ASMA ${ }^{* \star}$, SMA, S-100 protein, cytokeratin 14 , and vimentin were done in $8,6,8,14$, and 13 cases, respectively, due to restrains in paraffin-embedded tissue availability. 
two of two low grade spindle cell carcinomas with scattered squamous cells, and two of three MCBs with heterologous elements were positive for this marker.

\section{Vimentin}

Vimentin was evaluated in 13 cases. In the adjacencies of the neoplasms, vimentin decorated intralobular and perilobular stromal cells and vessel walls. Remarkably, vimentin was positive in all 13 cases evaluated in the current study.

\section{DISCUSSION}

The histogenesis of metaplastic breast carcinoma has challenged pathologists since the early days of diagnostic surgical pathology (1-10). In 1987 Oberman et al. (5) coined the designation "metaplastic carcinoma of the breast" as a term to describe a group of biphasic carcinomas of the mammary gland that are characterized by a mixture of glandular epithelial components, which frequently exhibit features of squamous differentiation, and mesenchymal malignant components with highly variable histologic features, including spindle cells, bone, cartilage, myxoid stroma, and anaplastic stroma with giant cells $(1,5-10)$.

Initially, MCBs were regarded as "collision tumors," but molecular genetic studies, by means of HUMARA clonality assays or by the finding of concurrent genetic alterations in glandular and mesenchymal cells, support a clonal ontogeny of the different components of MCBs (26-28).

Because MCBs are clonal tumors, the question that still remains unanswered is the origin of these neoplasms. Conflicting data have been published regarding the histogenesis and differentiation of MCB $(1,3,5,7,29-$ 32). Whereas some authors have favored a myoepithelial cell histogenesis $(1,5,7,29,30,31)$, others have refuted this hypothesis and have supported a secretory cell ontogeny for these neoplasms (3). Several lines of indirect evidence favors a putative myoepithelial histogenesis for MCBs, including the presence of cufflike proliferation of malignant cells around the residual breast ducts and metaplastic changes, such as squamous, chondroid, osteous, and mesenchymal-like metaplasia, which are usually observed in reactive myoepithelial cells and in myoepithelial cell tumors of the breast, salivary, and sweat glands $(1,29-31)$. Conversely, these phenomena are exceedingly rare in epithelial secretory cells of the breast. In addition, ultrastructural studies have also disclosed the presence of myoepithelial features in bona fide cases of MCB (32).

The current study brings new evidence for a myoepithelial cell origin in this heterogeneous group of neoplasms. We observed maspin expression in $93.7 \%$, p63 in $62.5 \%$, and P-cadherin in $57.1 \%$ of MCB cases, re- gardless of their histologic appearance. All cases were positive for at least one of these markers.

There are compelling data that militates that maspin may be used as a reliable myoepithelial marker (15, $16,25)$, because it consistently decorates nonneoplastic and neoplastic myoepithelial cells and, at variance, only rarely stains nonneoplastic or neoplastic secretory cells $(14-16,25)$.

Similar results regarding p63 expression were observed by Barbareschi et al. (18) in a series of 300 invasive carcinomas of different histotypes (18). These authors found that p63 expression was restricted to the adenoid-cystic carcinomas, metaplastic carcinomas with squamous metaplasia, and $4.6 \%$ of ductal carcinomas not otherwise specified (18). Wang et al. (28) also evaluated p63 expression in a unique case of metaplastic breast carcinoma that coexhibited squamous and cartilaginous metaplastic components; in this case, p63 expression was restricted to the squamous component. Our results corroborate those observed by Barbareschi et al. (18) and Wang et al. (28); we observed p63 expression in two of three cases of MCB with squamous metaplasia. A previously unreported finding of the current study is that p63 was strongly expressed in the nuclei of neoplastic spindle cells in low grade spindle MCB with or without squamous metaplasia (Fig. 2C). In our view, further studies are needed to evaluate the putative role of this marker in immunohistochemical panels designed for the differentiation between spindle cell MCB and other spindle cell lesions of the breast.

A large amount of data has been published in the last few years addressing P-cadherin expression in invasive and in situ breast carcinomas. In the different studies, P-cadherin expression ranged from $20 \%$ to $52 \%$ (1922,24 ) of invasive breast carcinomas, independent of the histologic type. P-cadherin-positive cases were associated with high proliferation rates, lack of estrogen and progesterone receptors, c-erb-B2 overexpression, p53 immunoreactivity, lymph node metastasis, and poor survival (19-22,24). Moreover, it has been shown that some special types of breast carcinomas are consistently immunoreactive for this marker, namely the metaplastic and medullary variants $(20,23)$. The biologic meaning of P-cadherin in breast neoplasms is not well understood (19-21,23). Peralta Soler et al. (24) and Gamallo et al. (20) raised the hypothesis that aberrant expression of $\mathrm{P}$-cadherin in breast cancer cells is associated with an embryonic phenotype similar to that of somatic stem cells $(20,22,24)$. Accordingly, P-cadherin expression was consistently observed in those samples included in the subgroup of breast carcinomas with basal and myoepithelial cell-like mRNA profile described by Perou et al. (11). Han et al. (23) reported P-cadherin expression in all cases of sarcomatoid MCB (spindle cell MCB) and carcinosarcoma (MCB with heterologous elements). Our 
study supports the finding of Han et al.(23) because two of three spindle cell MCBs and two of three MCBs with heterologous elements (carcinosarcomas) were positive for P-cadherin.

To further characterize whether tumors positive for one of the novel myoepithelial markers also showed a myoepithelial or stem (basal) cell phenotype, we also evaluated the expression of classic myoepithelial markers (ASMA in all cases and MSA and S-100 protein immunohistochemistry for selected cases because of restrictions in tissue availability). Because these classic markers are related to the smooth muscle apparatus and properties of myoepithelial cells, one would expect that myoepithelial cells, myoepithelial-derived tumors, and tumors with partial myoepithelial differentiation would express them, whereas undifferentiated stem (basal) cells and their tumors would not (18). We found a high frequency of classic myoepithelial markers expression in all histologic types of MCB. Our results are in accordance with the largest studies on MCB published to date, in which immunoreactivity for actin or S-100 protein was frequently observed in low and high grade spindle cell carcinomas $(2,7,32,33)$, matrix-producing MCB (6), and so-called carcinosarcomas $(8,34)$.

Cytokeratin 14 is an acidic cytokeratin that is positive in basal cells of stratified epithelia and myoepithelial cells of the breast and salivary glands (35). It is consistently expressed in squamous cell carcinomas (35), adenomyoepitheliomas of the breast (36), and myoepithelial tumors of the salivary glands $(35,36)$. Conversely, it is usually negative in most adenocarcinomas and especially in ductal and lobular carcinomas of the breast (35). Noteworthy, it has been advocated that $\mathrm{Ck} 14$ can be used to consistently support a diagnosis of myoepithelial cell tumors (35). We observed Ck14 expression in 11 of 14 cases of MCBs; thus, the balance of probabilities favors a myoepithelial or stem cell (basal cell) histogenesis or differentiation in MCBs.

Experimental data also support a myoepithelial cell histogenesis for MCB. Sapino et al.(37), using rat R3230AC mammary tumor-derived cell lines that display clones with epithelial and myoepithelial phenotypes, demonstrated that myoepithelial clone-derived tumors usually grew in a sarcomatous or carcinosarcomatous pattern, whereas epithelial-derived tumors presented a carcinomatous pattern (37). Altogether, these findings may rather support a myoepithelial phenotype instead of a basal or stem cell phenotype.

In conclusion, we reported a consistent expression of novel and classic myoepithelial markers in MCB. Although the entity of MCB encompasses a highly heterogeneous group of neoplasms, our findings and previously reported data support that most MCBs may have a myoepithelial histogenesis or harbor a myoepithelial differentiation.
Acknowledgments: The authors thank Marcello Fabiano Franco, M.D., Ph.D., Paulista Medical School, São Paulo, Brazil, for a critical review of the manuscript and Michal Michal, M.D., Sikl's Department of Pathology, Pilsen, Czech Republic, for his invaluable comments. The authors also thank Stuart Schnitt, M.D., Department of Pathology, Beth Israel Deaconess Medical Center, Boston, Massachusetts, for his insightful, constructive, and wise criticisms on our findings. The authors acknowledge the excellent technical assistance provided by Dina Leitão of IPATIMUP, Portugal.

\section{REFERENCES}

1. Tavassoli FA. Classification of metaplastic carcinomas of the breast. Pathol Annu 1992;27(pt 2):89-119.

2. Gobbi H, Simpson JF, Borowsky A, et al. Metaplastic breast tumors with a dominant fibromatosis-like phenotype have a high risk of local recurrence. Cancer 1999;85:2170-82.

3. Sneige N, Yaziji H, Mandavilli SR, et al. Low-grade (fibromatosislike) spindle cell carcinoma of the breast. Am J Surg Pathol 2001; 25:1009-16.

4. Chhieng C, Cranor M, Lesser ME, et al. Metaplastic carcinoma of the breast with osteocartilaginous heterologous elements. Am J Surg Pathol 1998;22:188-94.

5. Oberman HA. Metaplastic carcinoma of the breast. A clinicopathologic study of 29 patients. Am J Surg Pathol 1987;11:918-29.

6. Wargotz ES, Norris HJ. Metaplastic carcinomas of the breast. I. Matrix-producing carcinoma. Hum Pathol 1989;20:628-35.

7. Wargotz ES, Deos PH, Norris HJ. Metaplastic carcinomas of the breast. II. Spindle cell carcinoma. Hum Pathol 1989;20:732-40.

8. Wargotz ES, Norris HJ. Metaplastic carcinomas of the breast. III. Carcinosarcoma. Cancer 1989;64:1490-9.

9. Wargotz ES, Norris HJ. Metaplastic carcinomas of the breast. IV. Squamous cell carcinoma of ductal origin. Cancer 1990;65:272-6.

10. Wargotz ES, Norris HJ. Metaplastic carcinomas of the breast. V. Metaplastic carcinoma with osteoclastic giant cells. Hum Pathol 1990;21:1142-50.

11. Perou CM, Sorlie T, Eisen MB, et al. Molecular portraits of human breast tumours. Nature 2000;406:747-52.

12. Sorlie T, Perou CM, Tibshirani R, et al. Gene expression patterns of breast carcinomas distinguish tumor subclasses with clinical implications. Proc Natl Acad Sci U S A 2001;98:10869-74.

13. Hendrix MJ. De-mystifying the mechanism(s) of maspin. Nat Med 2000;6:374-6.

14. Maass N, Teffner M, Rösel F, et al. Decline in the expression of the serine proteinase inhibitor maspin is associated with tumour progression in ductal carcinomas of the breast. J Pathol 2001;195: 321-6.

15. Lele SM, Graves K, Gatalica Z. Immunohistochemical detection of maspin is a useful adjunct in distinguishing radial sclerosing lesion from tubular carcinoma of the breast. Appl Immunohistochem Molecul Morphol 2000;8: 32-6.

16. Reis Filho JS, Milanezi F, Silva P, et al. Maspin expression in myoepithelial tumours of the breast. Pathol Res Pract 2001;197: 817-21.

17. Reis-Filho JS, Schmidtt FC. Taking advantage of basic research: p63 is a reliable myoepithelial and stem cell marker. Adv Anat Pathol 2002;9:280-9.

18. Barbareschi M, Pecciarini L, Cangi MG, et al. p63, a p53 homologue, is a selective nuclear marker of myoepithelial cells of the human breast. Am J Surg Pathol 2001;25:1054-60.

19. Paredes J, Milanezi F, Viegas L, et al. P-cadherin expression is associated with high-grade ductal carcinoma in situ of the breast. Virchows Arch 2002;440:16-21.

20. Gamallo C, Moreno-Bueno G, Sarrio D, et al. The prognostic significance of P-cadherin in infiltrating ductal breast carcinoma. Mod Pathol 2001;14:650-4. 
21. Madhavan M, Srinivas P, Abraham E, et al. Cadherins as predictive markers of nodal metastasis in breast cancer. Mod Pathol 2001;14:423-7.

22. Palacios J, Benito N, Pizarro A, et al. Anomalous expression of P-cadherin in breast carcinoma. Correlation with E-cadherin expression and pathological features. Am J Pathol 1995;46:605-12.

23. Han AC, Soler AP, Knudsen KA, et al. Distinct cadherin profiles in special variant carcinomas and other tumors of the breast. Hum Pathol 1999;30:1035-9.

24. Peralta Soler A, Knudsen KA, Salazar H, et al. P-cadherin expression in breast carcinoma indicates poor survival. Cancer 1999;86: 1263-72.

25. Reis-Filho JS, Milanezi F, Schmitt FC. Maspin is expressed in the nuclei of breast myoepithelial cells. J Pathol 2002;197:272-3.

26. Zhuang Z, Lininger RA, Man YG, et al. Identical clonality of both components of mammary carcinosarcoma with differential loss of heterozygosity. Mod Pathol 1997;10:354-62.

27. Thompson L, Chang B, Barsky SH. Monoclonal origins of malignant mixed tumors (carcinosarcomas). Evidence for a divergent histogenesis. Am J Surg Pathol 1996;20:277-85.

28. Wang X, Mori I, Tang W, et al. Metaplastic carcinoma of the breast: p53 analysis identified the same point mutation in the three histologic components. Mod Pathol 2001;14:1183-6.

29. Hamperl H. The myothelia (myoepithelial cells). Normal state; regressive changes; hyperplasia; tumors. Curr Top Pathol 1970; 53:161-220.

30. Foote FW, Stewart FW. A histologic classification of carcinoma of the breast. Am J Cancer 1940;40:74-9.

31. Hamperl H. Über die Myothelien (myo-epithelialen Element) der Brustdrüse. Virchows Arch Path Anat 1939;305:171-215.

32. Harb JM, Komorowski RA, Vitali CM. Metaplastic breast carcinoma invading chest wall. Ultrastruct Pathol 1995;19:439-43.

33. Raju GC, Wee A. Spindle cell carcinoma of the breast. Histopathology 1990;16:497-9.

34. Simpson RH, Cope N, Skalova A, et al. Malignant adenomyoepithelioma of the breast with mixed osteogenic, spindle cell, and carcinomatous differentiation. Am J Surg Pathol 1998;22:631-6.

35. Chu PG, Lyda MH, Weiss LM. Cytokeratin 14 expression in epithelial neoplasms: a survey of 435 cases with emphasis on its value in differentiating squamous cell carcinomas from other epithelial tumors. Histopathology 2001;39:9-16.

36. Loose JH, Patchefsky AS, Hollander IJ, et al. Adenomyoepithelioma of the breast. A spectrum of biologic behavior. Am J Surg Pathol 1992;16:868-76.

37. Sapino A, Papotti M, Sanfilippo B, et al. Tumor types derived from epithelial and myoepithelial cell lines of R3230AC rat mammary carcinoma. Cancer Res 1992;52:1553-60. 